\title{
Multibaseline Polarimetric SAR Interferometry Coherence Optimization
}

\author{
Maxim Neumann, Student Member, IEEE, Laurent Ferro-Famil, Member, IEEE, and Andreas Reigber, Member, IEEE
}

\begin{abstract}
This letter analyzes different approaches for polarimetric optimization of multibaseline (MB) interferometric coherences. Two general methods are developed to simultaneously optimize coherences for more than two data sets. The first method provides every data set with a distinct dominant scattering mechanism (SM). The second optimization method is constrained to use equal SMs at all data sets. As the experimental results indicate, MB coherence optimization does improve the accuracy in the estimation of dominant SMs and the associated interferometric phases. Both methods are evaluated on real data acquired by the German Aerospace Agency (DLR)'s enhanced synthetic aperture radar sensor (ESAR) at L-band.
\end{abstract}

Index Terms-Coherence optimization, multibaseline (MB), polarimetric synthetic aperture radar interferometry (PolInSAR).

\section{INTRODUCTION}

$\mathbf{P}$ OLARIMETRIC synthetic aperture radar interferometry (PolInSAR) is an advanced technique that makes it possible to separate scattering centers inside a resolution cell. By varying the polarization states, optimal scattering mechanisms (SMs) with the highest coherence can be obtained. Optimizing coherence provides the possibility to reduce the uncertainty in interferometric phase estimation. The decorrelation terms are decreased with coherence optimization, and the signal-to-noise ratio is improved. This maximization makes the estimation of the topography more accurate. Polarimetric coherence optimization can also be applied to vertically structured media to resolve the dominant scattering centers.

With the introduction of PolInSAR, a coherence optimization technique was presented by Cloude and Papathanassiou [1]. This method is considered as the most general one since it allows different polarization states at the ends of the baseline. Colin et al. [2] outlined a general optimization routine with a constraint of equal polarization states. In the meantime, various other PolInSAR coherence optimization algorithms to optimize the coherence in a subspace and to give suboptimal solutions have been developed, for example, by Sagués et al. [3], Yamada et al. [4], Tabb et al. [5], Gomez-Dans and Quegan [6], and Qong [7].

The extension of single-baseline (SB) PolInSAR to multiple baselines increases the observation space. Analyzing a series of coherent polarimetric data sets enables more advanced and

Manuscript received October 10, 2006; revised August 3, 2007.

M. Neumann and L. Ferro-Famil are with the SAR Polarimetry Holography Interferometry Radargrammetry Team, Institute of Electronics and Telecommunications of Rennes, University of Rennes 1, 35042 Rennes, France (e-mail: maxn@cs.tu-berlin.de).

A. Reigber is with the Computer Vision and Remote Sensing Group, Berlin University of Technology, 10623 Berlin, Germany.

Color versions of one or more of the figures in this paper are available online at http://ieeexplore.ieee.org.

Digital Object Identifier 10.1109/LGRS.2007.908885 more accurate applications. The range varies from polarimetric differential interferometry (PolDInSAR) to parameter inversion techniques with more complex physical models. It is also of importance to identify the most coherent scattering center in all data sets, thus minimizing the phase errors. This delivers the most accurate estimates of the interferometric phases and of the SMs.

Given a multibaseline (MB) data set, coherences can be optimized independently for every baseline. This partly reproduces the results and errors of the SB case and leads to different dominant scattering centers depending on the chosen baseline. A better approach to find the most coherent and dominant scatterer is simultaneous optimization of MB coherences. This approach generally leads to lower coherence magnitudes, but the corresponding SMs and their interferometric phases are estimated on the basis of all available information and, thus, more accurately.

Most of the current airborne and spaceborne PolInSAR systems acquire data with spatial and temporal baseline separations. With respect to baseline separation properties, two coherence optimization criteria are considered. With proximate baselines, one would prefer to extract equal SMs (ESMs) of the highest coherence. With more divergent baseline properties, another approach is more reasonable, i.e., to use slightly different polarizations [multiple SMs (MSMs)] for different tracks to estimate the coherence of the dominant scatterers. As an example, changes in the scattering behavior, as caused, for example, by meteorological influences or vegetation growth between the acquisition times, will induce different polarization signatures for the same scattering structures. Both approaches, namely MSM and ESM, are analyzed, and two methods are developed and evaluated, as described in the following sections.

\section{MB POLINSAR}

An MB $n$-track geometry contains $(n / 2)(n-1)$ direct baselines. Fully polarimetric monostatic data can be represented in the Pauli basis, assuming reciprocity, for every track $i \in[1, n]$ by the scattering vector $\mathbf{k}_{i}$, i.e.,

$$
\mathbf{k}_{i}=\frac{1}{\sqrt{2}}\left[S_{i}^{\mathrm{HH}}+S_{i}^{\mathrm{VV}}, S_{i}^{\mathrm{HH}}-S_{i}^{\mathrm{VV}}, 2 S_{i}^{\mathrm{HV}}\right]^{T} .
$$

The MB PolInSAR coherency matrix $\mathbf{T}$, representing estimated covariance of polarimetric and interferometric channels, is generated by multilooking of the outer product of the aggregated scattering vector $\mathbf{k}$. Thus

$\mathbf{T}=\left\langle\mathbf{k k}^{\dagger}\right\rangle=\left[\begin{array}{ccc}\mathbf{T}_{11} & \cdots & \boldsymbol{\Omega}_{1 n} \\ \vdots & \ddots & \vdots \\ \boldsymbol{\Omega}_{1 n}^{\dagger} & \cdots & \mathbf{T}_{n n}\end{array}\right], \quad$ with $\quad \mathbf{k}=\left[\begin{array}{c}\mathbf{k}_{1} \\ \vdots \\ \mathbf{k}_{n}\end{array}\right]$ 
where \langle\rangle represents the multilooking operator, and $\dagger$ is the Hermitian transformation. $\mathbf{T}_{i i}$ contain polarimetric information, whereas $\boldsymbol{\Omega}_{i j}(i \neq j)$ contain baseline-dependent polarimetric and interferometric information.

The concept of SM is crucial to polarimetric interferometry and can be interpreted as representing the polarization state pair of the transmit and receive channels or as describing the physical characteristics of scatterers (hence the name). For the monostatic case, an SM vector corresponds to a complex unitary vector $\omega \in \mathbb{C}^{3}$ with four degrees of freedom. To obtain the scattering amplitude for a specific pair of transmit/receive polarizations, the scattering vector is projected onto the SM: $S_{i}=\boldsymbol{\omega}_{i}^{\dagger} \mathbf{k}_{i}$. SM vectors can be used to examine the covariance (cross correlation) of two interferometric data sets $i$ and $j$ for specific polarization states $\boldsymbol{\omega}_{i}$ and $\boldsymbol{\omega}_{j}: \sigma_{i j}=\left\langle S_{i} S_{j}^{*}\right\rangle=$ $\boldsymbol{\omega}_{i}^{\dagger} \boldsymbol{\Omega}_{i j} \boldsymbol{\omega}_{j}$. Typically, covariances are examined for equal polarizations utilizing the same SMs: $\boldsymbol{\omega}_{i}=\boldsymbol{\omega}_{j}$. The corresponding interferometric coherence and phase estimates, in the general case, are the following:

$\gamma_{i j\left(\boldsymbol{\omega}_{i}, \boldsymbol{\omega}_{j}\right)}=|\gamma| e^{i \phi}=\frac{\sigma_{i j}}{\sqrt{\sigma_{i i} \sigma_{j j}}}=\frac{\boldsymbol{\omega}_{i}^{\dagger} \boldsymbol{\Omega}_{i j} \boldsymbol{\omega}_{j}}{\sqrt{\boldsymbol{\omega}_{i}^{\dagger} \mathbf{T}_{i i} \boldsymbol{\omega}_{i} \boldsymbol{\omega}_{j}^{\dagger} \mathbf{T}_{j j} \boldsymbol{\omega}_{j}}}$.

\section{MB COHERENCE OPTIMIZATION}

In contrast with the independent SB coherence optimization methods, MB methods simultaneously optimize coherences in several baselines. Thus, they are expected to deliver more robust estimates of the optimized interferometric phases and dominant SMs.

The general MB-MSM method assigns a distinct SM to each track. This approach allows one to optimize the coherence for SMs that might have different polarimetric signatures in different data sets. The MB-ESM method, on the other hand, enforces equal polarimetric signatures of scatterers along all baselines $\left(\boldsymbol{\omega}_{i}=\boldsymbol{\omega}_{j} \forall i, j\right)$, and the application of this method is restricted to a single $\mathrm{SM}$ for the dominant scatterer.

The general MB optimization problem can thus be stated as the maximization of a function $f_{\left(\boldsymbol{\omega}_{1}, \ldots, \boldsymbol{\omega}_{n}\right)}$ (possibly $\boldsymbol{\omega}_{i}=\boldsymbol{\omega}_{j}$ for ESM) incorporating coherence moduli for all baselines. In the simplest case, the optimization function $f$ is determined by the sum over coherence moduli: $f=\sum\left|\gamma_{i j}\right|$. As can be shown, there are no exact analytical methods applicable for the simple sum optimization problem, neither for MSM nor for ESM. However, two algorithms will be presented that achieve such optimizations with a high degree of accuracy and efficiency.

\section{A. MSM Optimization}

The SB coherence optimization with two SMs (i.e., SB-MSM) [1], which is a canonical correlation analysis (CCA) problem [8], optimizes the modulus of the covariance $\boldsymbol{\omega}_{i}^{\dagger} \boldsymbol{\Omega}_{i j} \boldsymbol{\omega}_{j}$ for two data sets $i$ and $j$ while keeping the variances $\boldsymbol{\omega}_{i}^{\dagger} \mathbf{T}_{i i} \boldsymbol{\omega}_{i}$ and $\boldsymbol{\omega}_{j}^{\dagger} \mathbf{T}_{j j} \boldsymbol{\omega}_{j}$ constant. In [1], the modulus of the complex Lagrangian $L$ is maximized by introducing the real-valued multipliers $\lambda_{i}$ and $\lambda_{j}$, i.e.,

$$
L=\boldsymbol{\omega}_{i}^{\dagger} \boldsymbol{\Omega}_{i j} \boldsymbol{\omega}_{j}-\lambda_{i}\left(\boldsymbol{\omega}_{i}^{\dagger} \mathbf{T}_{i i} \boldsymbol{\omega}_{i}-1\right)-\lambda_{j}\left(\boldsymbol{\omega}_{j}^{\dagger} \mathbf{T}_{j j} \boldsymbol{\omega}_{j}-1\right)
$$

As it has been shown, the solution can be obtained by setting the partial derivatives of $L$ with respect to variables $\lambda_{i}, \lambda_{j}, \boldsymbol{\omega}_{i}$, and $\omega_{j}$ to zero. Thus

$$
\begin{aligned}
& \frac{\partial L}{\partial \lambda_{i}}=\boldsymbol{\omega}_{i}^{\dagger} \mathbf{T}_{i i} \boldsymbol{\omega}_{i}-1=0 \\
& \frac{\partial L}{\partial \lambda_{j}}=\boldsymbol{\omega}_{j}^{\dagger} \mathbf{T}_{j j} \boldsymbol{\omega}_{j}-1=0 \\
& \frac{\partial L}{\partial \boldsymbol{\omega}_{i}^{\dagger}}=\boldsymbol{\Omega}_{i j} \boldsymbol{\omega}_{j}-\lambda_{i} \mathbf{T}_{i i} \boldsymbol{\omega}_{i}=0 \\
& \frac{\partial L^{\dagger}}{\partial \boldsymbol{\omega}_{j}^{\dagger}}=\boldsymbol{\Omega}_{i j}^{\dagger} \boldsymbol{\omega}_{i}-\lambda_{j} \mathbf{T}_{j j} \boldsymbol{\omega}_{j}=0 .
\end{aligned}
$$

Optimal SMs and the corresponding coherences are obtained from the resulting eigenvalue problems, i.e.,

$$
\begin{aligned}
& \mathbf{T}_{j j}^{-1} \boldsymbol{\Omega}_{i j}^{\dagger} \mathbf{T}_{i i}^{-1} \boldsymbol{\Omega}_{i j} \boldsymbol{\omega}_{j}=\lambda_{i} \lambda_{j} \boldsymbol{\omega}_{j} \\
& \mathbf{T}_{i i}^{-1} \boldsymbol{\Omega}_{i j} \mathbf{T}_{j j}^{-1} \boldsymbol{\Omega}_{i j}^{\dagger} \boldsymbol{\omega}_{i}=\lambda_{i} \lambda_{j} \boldsymbol{\omega}_{i}
\end{aligned}
$$

Since $\mathbf{T}_{i i}$ and $\mathbf{T}_{j j}$ are Hermitian and positive definite, the inverses, as well as square roots, of these matrices exist. One can show that $\lambda_{i}=\lambda_{j}=\lambda$ by left multiplying (7) and (8) with $\boldsymbol{\omega}_{i}^{\dagger}$ and $\boldsymbol{\omega}_{j}^{\dagger}$ and using (5) and (6). Furthermore, the complex Lagrange function (4) can be transformed into a real-valued function. These two modifications make it possible to express the optimization problem in the following notation:

$$
L=\sum_{i=1}^{n} \sum_{j=1 \neq i}^{n} \boldsymbol{\omega}_{i}^{\dagger} \boldsymbol{\Omega}_{i j} \boldsymbol{\omega}_{j}-\lambda \sum_{i=1}^{n}\left(\boldsymbol{\omega}_{i}^{\dagger} \mathbf{T}_{i i} \boldsymbol{\omega}_{i}-1\right) .
$$

This function is real valued due to the fact that the first term of $L$ stands for the sum of $\operatorname{Re}\left(\boldsymbol{\omega}_{i}^{\dagger} \boldsymbol{\Omega}_{i j} \boldsymbol{\omega}_{j}\right)$ for all $i \neq j$, since $\boldsymbol{\omega}_{i}^{\dagger} \boldsymbol{\Omega}_{i j} \boldsymbol{\omega}_{j}=\left(\boldsymbol{\omega}_{j}^{\dagger} \boldsymbol{\Omega}_{j i} \boldsymbol{\omega}_{i}\right)^{\dagger}$. This modification uses the phase ambiguity between $\boldsymbol{\omega}_{i}$ and $\boldsymbol{\omega}_{j}$, i.e., $\arg \left(\boldsymbol{\omega}_{i}^{\dagger} \boldsymbol{\omega}_{j}\right)$, to cause a shift of coherence phases toward zero. The second term in (11) is real valued since it contains quadratic forms of Hermitian matrices. For $n=2$, the optimization problem formulated in (11) is equivalent to (4), i.e., to the SB case.

When more than two data sets are taken into consideration, optimization is referred to as the multiset CCA [9]. In this case, (11) can be interpreted as a weighted optimization of coherences since it refers to optimizing the sum of covariances $\boldsymbol{\omega}_{i}^{\dagger} \boldsymbol{\Omega}_{i j} \boldsymbol{\omega}_{j}$ while keeping the sum of variances $\boldsymbol{\omega}_{i}^{\dagger} \mathbf{T}_{i i} \boldsymbol{\omega}_{i}$ constant. Setting the partial derivatives of (11) to zero will lead to the generalized eigenvalue problem (12) that optimizes a linear combination of coherences $(i \in[1, \ldots, n])$, i.e.,

$$
\begin{aligned}
& \sum_{j=1 \neq i}^{n} \boldsymbol{\Omega}_{i j} \boldsymbol{\omega}_{j}=\lambda \mathbf{T}_{i i} \boldsymbol{\omega}_{i} \Longleftrightarrow \mathbf{A} \boldsymbol{\omega}=\lambda \mathbf{B} \boldsymbol{\omega} \Longleftrightarrow \\
& {\left[\begin{array}{cccc}
\mathbf{0} & \boldsymbol{\Omega}_{12} & \cdots & \boldsymbol{\Omega}_{1 n} \\
\boldsymbol{\Omega}_{21} & \mathbf{0} & \cdots & \boldsymbol{\Omega}_{2 n} \\
\vdots & \vdots & \ddots & \vdots \\
\boldsymbol{\Omega}_{n 1} & \boldsymbol{\Omega}_{n 2} & \cdots & \mathbf{0}
\end{array}\right]\left[\begin{array}{c}
\boldsymbol{\omega}_{1} \\
\boldsymbol{\omega}_{2} \\
\vdots \\
\boldsymbol{\omega}_{n}
\end{array}\right]=\lambda\left[\begin{array}{cccc}
\mathbf{T}_{11} & \mathbf{0} & \cdots & \mathbf{0} \\
\mathbf{0} & \mathbf{T}_{22} & \cdots & \mathbf{0} \\
\vdots & \vdots & \ddots & \vdots \\
\mathbf{0} & \mathbf{0} & \cdots & \mathbf{T}_{n n}
\end{array}\right]\left[\begin{array}{c}
\boldsymbol{\omega}_{1} \\
\boldsymbol{\omega}_{2} \\
\vdots \\
\boldsymbol{\omega}_{n}
\end{array}\right] .}
\end{aligned}
$$


The optimized coherence modulus for the SB case is equal to the largest eigenvalue in (12) and the square root of the largest eigenvalues in (9) and (10). Furthermore, the phase of the SB optimized coherence is equal to $-\arg \left(\boldsymbol{\omega}_{1}^{\dagger} \boldsymbol{\omega}_{2}\right)\left(=\arg \left(\boldsymbol{\omega}_{2}^{\dagger} \boldsymbol{\omega}_{1}\right)\right)$ from (12) since (11) automatically deals with the phase ambiguity issue. For more than two data sets, the largest eigenvalue does not anymore correspond to the optimized coherence modulus, but rather to the weighted mean of the optimized coherence moduli. However, the eigenvectors include the optimal SMs, although unnormalized and containing phase ambiguities. Both issues, if not removed, might distort the interpretation of optimal SMs and interferometric phases.

MB-MSM coherence optimization can be summarized by the algorithm that follows.

1) Obtain eigenvectors from the generalized eigenvalue problem (12): $\mathbf{A} \boldsymbol{\omega}=\lambda \mathbf{B} \boldsymbol{\omega}$, where $\mathbf{A}=\mathbf{T}-\mathbf{B}, \mathbf{B}=$ $\bigoplus_{i=1}^{n} \mathbf{T}_{i i}$, and $\bigoplus$ is the direct sum operator.

2) Normalize the SM vectors from $\boldsymbol{\omega}=\left[\boldsymbol{\omega}_{1}, \ldots, \boldsymbol{\omega}_{n}\right]^{T}$, so that for all $i \in[1, n]: \boldsymbol{\omega}_{i}^{\dagger} \boldsymbol{\omega}_{i}=1$.

3) Remove the phase shift from these vectors with respect to an arbitrary track $m \in[1, n]$, so that for all $i \in[1, n]$ : $\arg \left(\boldsymbol{\omega}_{m}^{\dagger} \boldsymbol{\omega}_{i}\right)=0$.

4) Compute, if needed, optimized coherences according to (3) using vectors $\boldsymbol{\omega}_{i}$ after normalization and the removal of phase ambiguities.

\section{B. ESM Optimization}

For relatively small temporal and spatial separations between data sets, polarimetric coherency matrices $\mathbf{T}_{i i}$ are often very similar. Allowing different SMs for all data sets becomes less important or even undesirable. In [2], an optimization method, which constrains the optimized SMs to be equal (SB-ESM), is presented for the SB case. It is based on the numerical range [10] properties of the matrix $\Pi_{i j}$, i.e.,

$$
\boldsymbol{\Pi}_{i j}=\mathbf{T}_{e}^{-1 / 2} \boldsymbol{\Omega}_{i j} \mathbf{T}_{e}^{-1 / 2}, \quad \text { where } \quad \mathbf{T}_{e}=\frac{1}{n} \sum_{i=1}^{n} \mathbf{T}_{i i} .
$$

For the SB case, as in [2], $i=1, j=2$, and $n=2$. The numerical range of matrix $\Pi_{i j}, \mathcal{W}\left(\Pi_{i j}\right)$, can be interpreted as the set of coherences $\widetilde{\gamma}$ of $\boldsymbol{\Pi}_{i j}$ in all polarizations, i.e.,

$$
\begin{gathered}
\widetilde{\gamma}_{i j(\mathbf{w})}=\mathbf{w}^{\dagger} \boldsymbol{\Pi}_{i j} \mathbf{w} \quad \mathbf{w}=\frac{\sqrt{\mathbf{T}_{e}} \boldsymbol{\omega}}{\boldsymbol{\omega}^{\dagger} \sqrt{\mathbf{T}_{e} \boldsymbol{\omega}}} \\
\mathcal{W}\left(\boldsymbol{\Pi}_{i j}\right)=\left\{\mathbf{x}^{\dagger} \boldsymbol{\Pi}_{i j} \mathbf{x}: \mathbf{x} \in \mathbb{C}^{3}, \mathbf{x}^{\dagger} \mathbf{x}=1\right\} .
\end{gathered}
$$

The maximal coherence modulus of $\Pi_{i j}$ corresponds to the numerical radius $r\left(\boldsymbol{\Pi}_{i j}\right)$, i.e.,

$$
r\left(\boldsymbol{\Pi}_{i j}\right)=\max \left\{\left|\mathbf{x}^{\dagger} \boldsymbol{\Pi}_{i j} \mathbf{x}\right|: \mathbf{x} \in \mathbb{C}^{3}, \mathbf{x}^{\dagger} \mathbf{x}=1\right\} .
$$

In [2], an iterative method [11] is used to compute $r\left(\boldsymbol{\Pi}_{i j}\right)$ for the SB case.

The remainder of this section describes an extension of the SB algorithm to the MB case (MB-ESM). The sum of coherences $\sum\left|\widetilde{\gamma}_{i j(\mathbf{w})}\right|$ is taken as the optimization criterion, where the SMs are all equal: $\mathbf{w}_{i}=\mathbf{w}_{j}=\mathbf{w}$ for all $i, j \in[1, n]$.
In an attempt to remove the modulus operation, the phase shift variables $\theta_{i j} \in[-\pi, \pi], \theta_{j i}=-\theta_{i j}$, are introduced to validate the following inequality:

$$
\max _{\mathbf{w}} \sum_{i=1}^{n} \sum_{j=1 \neq i}^{n} \widetilde{\gamma}_{i j(\mathbf{w})} e^{-i \theta_{i j}} \leq \max _{\mathbf{w}} \sum_{i=1}^{n} \sum_{j=1 \neq i}^{n}\left|\widetilde{\gamma}_{i j(\mathbf{w})}\right|
$$

The left term is real valued since $\widetilde{\gamma}_{i j(\mathbf{w})} e^{-i \theta_{i j}}=$ $\left(\widetilde{\gamma}_{j i(\mathbf{w})} e^{-i \theta_{j i}}\right)^{\dagger}$, as was the case with (11). The maximum of the left side depends on the given set of phase shift variables $\left\{\theta_{i j}\right\}$, whereas the maximum on the right side is constant. Equality is achieved when phase shifts are equal to the phases of optimal coherences, so that the real parts of the phase-shifted optimal coherences are equal to the coherence moduli. Therefore, the optimization process consists in the simultaneous search for the optimized coherence phases and the corresponding optimal SM. As for the SB approach, the MB-ESM coherence optimization is not analytically solvable. However, an efficient iterative optimization method is presented, which converges in a few (i.e., two to five) iterations.

An estimate for the optimal SM can be obtained from the eigenvector associated with the largest eigenvalue of the combined Hermitian matrix $\mathbf{H}$ in

$$
\mathbf{H w}=\lambda \mathbf{w}, \quad \text { where } \quad \mathbf{H}=\sum_{i=1}^{n} \sum_{j=1 \neq i}^{n} \boldsymbol{\Pi}_{i j} e^{-i \theta_{i j}} .
$$

Estimates for the optimal phase shifts are in turn obtained from

$$
\theta_{i j}=\arg \left(\mathbf{w}^{\dagger} \boldsymbol{\Pi}_{i j} \mathbf{w}\right)
$$

The phase shifts can then be reintroduced to (18) to obtain an improved estimate of the optimal SM w. By iteratively adjusting the phase shifts $\theta_{i j}$, one obtains progressively better estimates of $\mathbf{w}$.

This method may lead to a suboptimal local maximum. To avoid this, one can perform several optimizations with different initial phase shifts. Another approach is an effective strategy for initialization. Since ESM coherence sets describe simple convex filled regions in the unitary complex coherence plane [2], phase shift angles $\theta_{i j}$ can be initialized with the trace phases of $\Pi_{i j}$. Such an initialization significantly improves the optimization performance with respect to the number of iterations and robustness.

It should be noted that when the polarimetric coherency matrices $\mathbf{T}_{i i}$ significantly differ, the ESM coherence optimization might fail to converge on the global optimum. In such cases, the MSM coherence optimization method should be preferred.

Finally, the steps involved in the proposed MB-ESM coherence optimization algorithm are given here.

1) Initialization: $\theta_{i j}=\arg \left(\operatorname{trace} \Pi_{i j}\right) ; \hat{\lambda}=0$.

2) Computation of $\mathbf{H}$ and $\mathbf{w}$ from (18) with current estimates of optimal phase shifts $\theta_{i j}$. w is the eigenvector corresponding to the highest eigenvalue $\lambda_{\max }$.

3) Improved estimation of $\theta_{i j}$ using computed w via (19). 


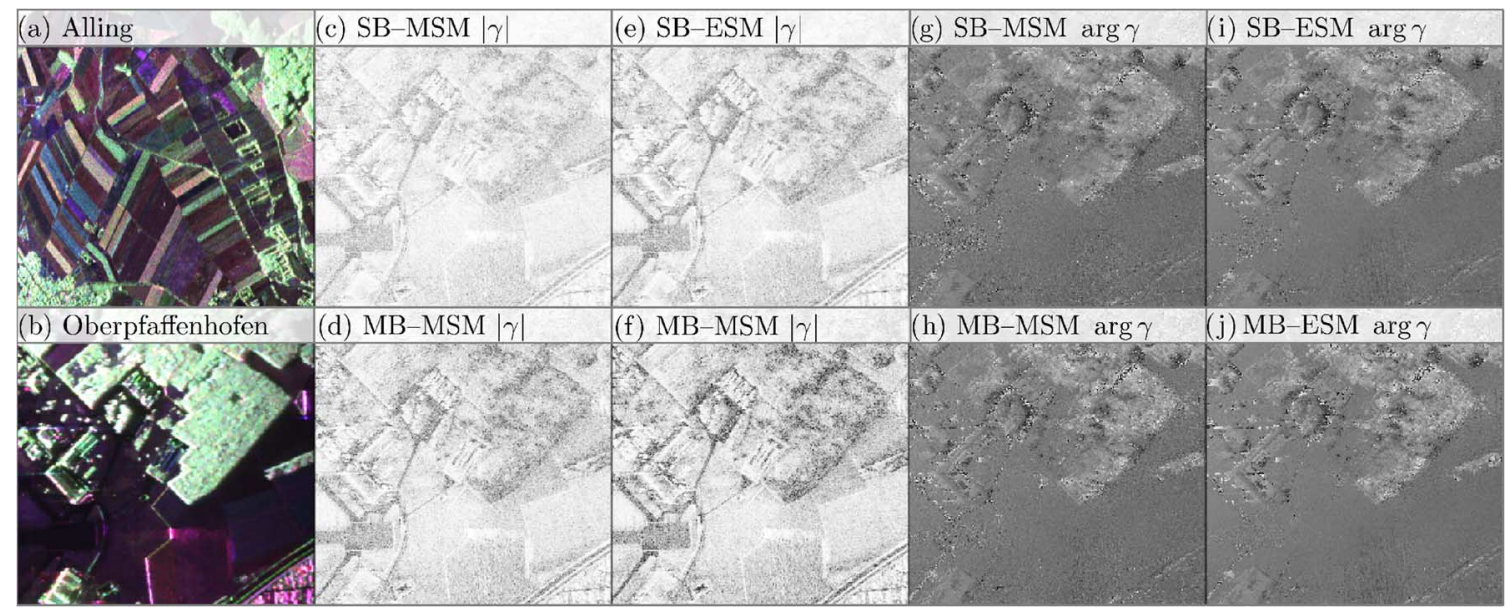

Fig. 1. Experimental scenes. (a) and (b) Alling and Oberpfafenhoffen areas in Pauli decomposition. (c)-(f) Optimized coherence magnitudes $\left|\gamma_{12}\right|$ for (b) of the first baseline for SB-MSM, SB-ESM, MB-MSM, and MB-ESM. (g)-(j) Optimized coherence phases arg $\gamma_{12}$ of (b) of the first baseline for SB-MSM, SB-ESM, MB-MSM, and MB-ESM.

4) Termination criterion: $\lambda_{\max }-\hat{\lambda} \leq \varepsilon$, where $\varepsilon$ is an arbitrary small constant. If the criterion is not met, then $\hat{\lambda}=\lambda_{\max }$, and go to step 2).

5) The optimal SM vector $\boldsymbol{\omega}$ and corresponding optimal coherences are calculated from $\mathbf{w}$ via $\boldsymbol{\omega}=$ $\mathbf{T}_{e}^{-1 / 2} \mathbf{w} /\left(\mathbf{w}^{\dagger} \mathbf{T}_{e}^{-1 / 2} \mathbf{w}\right)$ and (14).

\section{EXPERIMENTAL RESUlTS AND DISCUSSION}

The performance of optimization methods greatly depends on the configuration of the acquisition system (e.g., baseline lengths, acquisition times, and frequency) and the imaged media. The applicability is limited by the critical spatial and temporal separations between the data sets, and in case of strong decorrelation in most data sets, the obtained results might be questionable. Evaluation experiments on real data are presented to emphasize the difference and the sensitivity of the proposed MB coherence optimization methods versus the SB coherence optimization methods.

Two scenes have been examined. The first scene is a rural area near Alling, Germany, and is shown in Fig. 1(a). This area contains mostly vegetated agricultural fields. The data set comprises three coherent tracks with spatial baselines between 1 and $40 \mathrm{~m}$ and temporal baselines of 15 and $30 \mathrm{~min}$. Another scene in the Oberpfaffenhofen area [Fig. 1(b)] contains diverse scattering media, including forests, surface, and urban areas. Five tracks with baselines between 5 and $37 \mathrm{~m}$ and temporal separations between $15 \mathrm{~min}$ and $1 \mathrm{~h}$ are used. The same preprocessing procedures have been applied to both data sets, including flat-earth removal, range spectral filtering, and multilooking with 16 looks. Multilooking was done by spatial summation, not smoothing, to ensure a high degree of statistical independence between neighboring samples.

Fig. 1(c)-(f) and (g)-(j) shows the coherence moduli and coherence phases of the Oberpfaffenhofen scene after optimization with SB-MSM, SB-ESM, MB-MSM, and MB-ESM methods, respectively, for the first baseline. The corresponding baseline has a 5-m spatial separation and a 15-min temporal separation. The modulus images provide visual evidence that SB optimized coherences achieve higher values than their MB counterparts. The decrease of coherence moduli for MB coher-
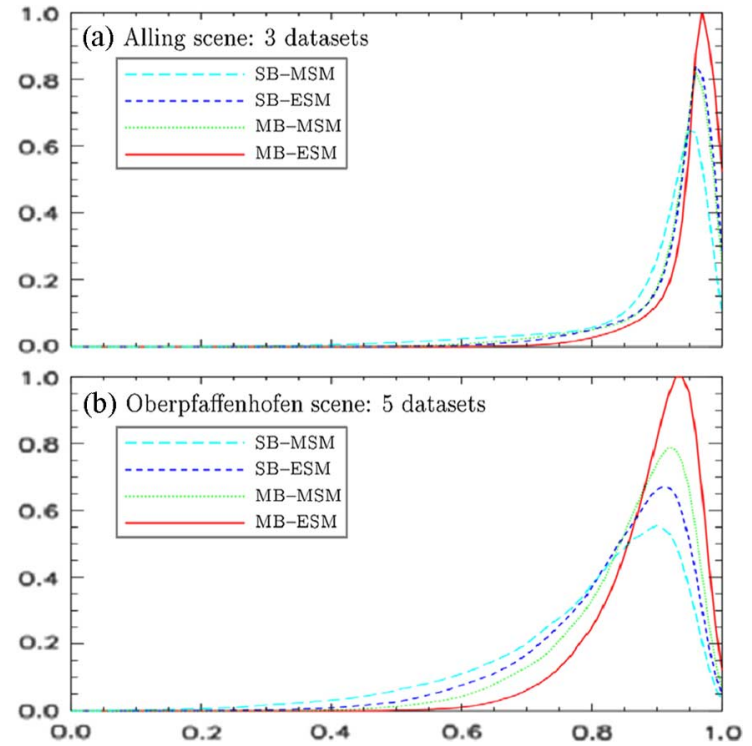

Fig. 2. Normalized optimal coherence moduli histograms. (a) Alling scene: agricultural region with three data sets. (b) Oberpfafenhoffen scene: forested region with five data sets.

ence optimization can also be observed on coherence moduli histograms for the given scenes in Fig. 2. This is related to the number of constraints and the dimension of the available search space. The contrast improvement of MB optimization techniques is conspicuous, particularly over forested areas. This tendency might be interpreted as the reduction of optimal coherence bias [12]. The advantage in utilizing numerous baselines for the optimization lies here: by interferometric baseline-dependent multilooking, i.e., by deliberately choosing suitable baselines, the optimal coherence bias might be reduced. However, further experiments have to be carried out to evaluate the bias reduction possibilities of MB optimizaton techniques.

It is important to note the major difference between the SB and the MB methods. Since the SB methods independently optimize coherences, they acquire higher coherence values, but the corresponding phases and SMs are different, indicating different dominant scattering centers. Such effects can be more 


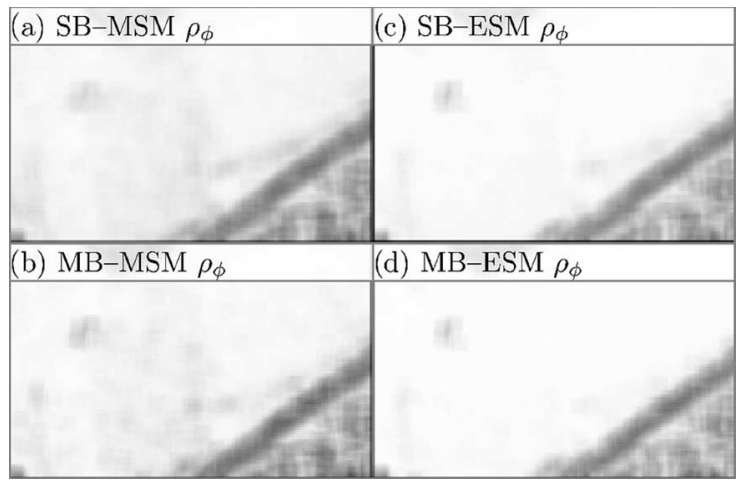

Fig. 3. Local correlation $\rho_{\phi}$ of optimized interferometric phases with $N=$ $7 \times 7=49$ over all baselines.

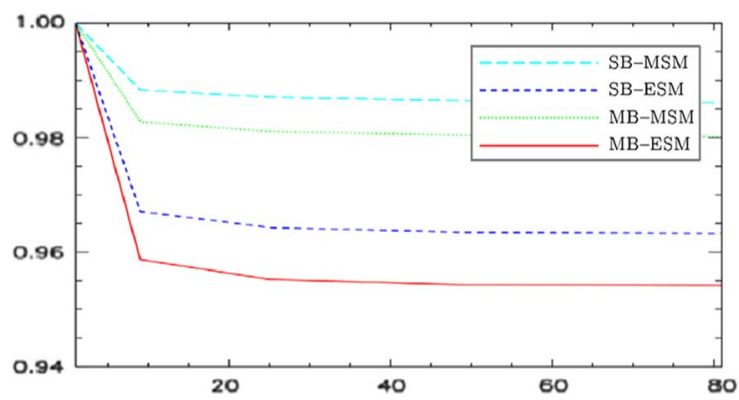

Fig. 4. Mean of $\rho_{\phi}$ as a function of spatial neighborhood averaging $N$.

closely examined by considering the interferometric phases of optimized coherences and their spatial variances. The differences of optimized coherence phases in Fig. 1(g)-(j) are hardly visible. However, quantitative examination of the local phase correlation over homogeneous areas reveals the improvement of the phase stability with a higher number of baselines. The correlation of the optimized phases $\rho_{\phi}$ is computed from the phase $\phi$ of the optimized coherence in a local window of $N$ samples, i.e.,

$\rho_{\phi}=\left|\left\langle e^{i \phi}\right\rangle\right|=\left|\frac{1}{N} \sum_{j=1}^{N} e^{i \phi_{j}}\right|, \quad$ where $\quad \phi_{j}=\arg \gamma_{j}^{\mathrm{opt}}$

An area of fields in the lower right edge of the Oberpfaffenhofen test site has been examined. This area is assumed to be homogeneous and devoid of topographic variations. Fig. 3 shows the local phase correlation $\rho_{\phi}$ for different optimization methods using a $7 \times 7$ averaging window. The averaged correlation for all baselines is presented. The diagram in Fig. 4 presents the results of the mean correlation coefficient calculations on homogeneous fields and for different averaging windows. These results demonstrate the improvement of the phase stability of simultaneous MB coherence optimization methods for both MSM and ESM.

\section{CONCLUSION}

MB coherence optimization concepts have been analyzed, and two optimization algorithms have been developed for the most general cases: one with MSMs in the data sets and one with ESMs. Initial experiments regarding MB coherence optimization properties have been conducted and discussed.

It has been observed that even when enforcing simultaneous MB coherence optimization, it is still possible to achieve very high coherences, coming close to SB optimized coherences. It has been shown that the utilization of multiple baselines for the optimization of coherences has certain advantages over SB optimization with regard to accuracy improvement in the estimation of dominant coherent phase centers and SMs, and coherence contrast improvement, which may be related to the optimal coherence bias reduction.

The extension of polarimetric optimization to the MB case has potential practical uses in various areas. The presented methods provide coherence optimization techniques to applications with more than one baseline for the first time. New fields are, for instance, PolDInSAR, permanent scatterer determination, classification, or change monitoring and detection over multitemporal and MB data sets. Furthermore, utilizing more than two data sets, if available, will improve the robustness in determining the most coherent SMs and is useful even for SB applications, such as digital elevation model extraction or parameter inversion.

\section{ACKNOWLEDGMENT}

The authors would like to thank M. Jäger and the anonymous reviewers for the constructive comments and corrections that helped to improve this letter.

\section{REFERENCES}

[1] S. R. Cloude and K. P. Papathanassiou, "Polarimetric optimization in radar interferometry," Electron. Lett., vol. 33, no. 13, pp. 1176-1178, Jun. 1997.

[2] E. Colin, C. Titin-Schnaider, and W. Tabbara, "An interferometric coherence optimization method in radar polarimetry for high-resolution imagery," IEEE Trans. Geosci. Remote Sens., vol. 44, no. 1, pp. 167-175, Jan. 2006.

[3] L. Sagués, J. M. Lopez-Sanchez, J. Fortuny, X. Fabregas, A. Broquetas, and A. J. Sieber, "Indoor experiments on polarimetric SAR interferometry," IEEE Trans. Geosci. Remote Sens., vol. 38, no. 2, pp. 671-684, Mar. 2000

[4] H. Yamada, Y. Yamaguchi, Y. Kim, E. Rodriguez, and W. M. Boerner, "Polarimetric SAR interferometry for forest analysis based on the ESPRIT algorithm," IEICE Trans. Electron., vol. E84-C, no. 12, pp. 1917-1924, Dec. 2001.

[5] M. Tabb, J. Orrey, T. Flynn, and R. Carande, "Phase diversity: A decomposition for vegetation parameter estimation using polarimetric SAR interferometry," in Proc. EUSAR, Cologne, Germany, Jun. 2002, pp. 721-724.

[6] J. L. Gomez-Dans and S. Quegan, "Constraint coherence optimisation in polarimetric interferometry of layered targets," in Proc. POLINSAR, Frascati, Italy, Jan. 2005.

[7] M. Qong, "Coherence optimization using the polarization state conformation in PolInSAR," IEEE Geosci. Remote Sens. Lett., vol. 2, no. 3, pp. 301-305, Jul. 2005.

[8] H. Hotelling, "Relations between two sets of variates," Biometrika, vol. 28, no. 3/4, pp. 321-377, Dec. 1936.

[9] J. R. Kettenring, "Canonical analysis of several sets of variables," Biometrika, vol. 58, no. 3, pp. 433-451, Dec. 1971.

[10] R. A. Horn and C. R. Johnson, Topics in Matrix Analysis. Cambridge, U.K.: Cambridge Univ. Press, 1991.

[11] G. A. Watson, "Computing the numerical radius," Linear Algebra Appl., vol. 234, pp. 163-172, Feb. 1996.

[12] R. Fomena and S. Cloude, "On the role of coherence optimisation in polarimetric SAR interferometry," in Proc. CEOS SAR CAL/VAL Workshop, Adelaide, Australia, Sep. 2005. 